\title{
$\begin{array}{ll}\text { Research Square } & \text { Preprints are preliminary reports that have not undergone peer review. } \\ \text { They should not be considered conclusive, used to inform clinical practice, } \\ \text { or referenced by the media as validated information. }\end{array}$
}

\section{The Effect of Extracorporeal Shock Wave on Osteonecrosis of Femoral Head: A Systematic Review and Meta-Analysis}

Jin Mei ( $\nabla$ meijin@stu.cdutcm.edu.cn )

Chengdu University of Traditional Chinese Medicine Affiliated Hospital https://orcid.org/0000-0002-

7446-3329

\section{Lili Pang}

Chengdu University of Traditional Chinese Medicine Affiliated Hospital

\section{Yu Gong}

Hunan Provincial People's Hospital

\section{Zhongchao Jiang}

Chengdu University of Traditional Chinese Medicine Affiliated Hospital

\section{Research article}

Keywords: Extracorporeal shock wave $₫$ Osteoncerosis of femoral head, Meta-analysis, Harris hip score, Pain score

Posted Date: June 22nd, 2020

DOI: https://doi.org/10.21203/rs.3.rs-37193/v1

License: (c) (1) This work is licensed under a Creative Commons Attribution 4.0 International License. Read Full License 


\section{Abstract}

Background: This study aimed to determine whether ESWT (extracorporeal shock wave therapy) has an efficient effect on ONFH (osteonecrosis of femoral head) in clinical outcomes and radiography outcomes.

Method: Two authors independently searched the papers on the treatment of femoral head necrosis with extracorporeal shock wave in CNKI (China National Knowledge Infrastructure), VIP (China Science and Technology Journal Database), CSPD (China Science Periodical Database) , Pubmed, Embase and Springer databases. Search period from the inception dates to June 2, 2020 and have no limitations in language; Two authors independently conducted Quality evaluation and data extraction for included studies and preformed a meta-analysis with data extracted and calculated by using review manager 5.3.

Result: Nine articles with 409 patients were included in this meta-analysis. The pooled results of HHS (Harris hip score) in eight studies with 337 hips show that ESWT achieved higher Harris scores compared to before treatment $(\mathrm{MD}=-19.95 ; 95 \% \mathrm{Cl}:-26.27,-13.64 ;)$. The differences were statistically significant $(p<0.01)$. The pooled results of VAS (visual analogue score) in seven studies with 253 hips show that ESWT achieved lower VAS compared to baseline (MD $=2.77 ; 95 \% \mathrm{Cl}: 1.88,3.65$;), the differences were statistically significant $(p<0.01)$. the pooled results of lesion of MRI with 164 hips show that ESWT decrease the lesion area of MRI (SMD=1.03,Cl: $0.75,1.30$,),the differences were statistically significant $(p<0.01)$.

Conclusion: ESWT have an effect in pain relief and can improve motion function. It's effect better than surgical groups (core decompression and core decompression with bone grafting). But it may not decrease the lesion area of the femoral head on MRI.

\section{Background}

ONFH is also known as avascular necrosis of the femoral head (AVNFH) or aseptic necrosis of the femoral head (ANFH)[1]. About 20,000 to 30,000 new patients are diagnosed with ONFH per year $\mathrm{n}$ the United States[2]. And approximately 2200 additional patients would diagnose with ONFH annually in Japan[3]. The pain of the patient in the deep groin is the most common symptom and influence the knee or ipsilateral buttock[4]. Degenerative changes of the hip joint and the collapse of the femoral head and degenerative changes of the hip joint occur later in the disease. It is important for young patients in precollapse stage to keep the femoral head intact considering the limited period of the use of prosthesis. So the early intervention to ESWT may be an important treatment approach. Current treatment of hip preservation include core decompression, nonvascularized or vascularized bone-grafting, rotational osteotomy, and muscle pedicle grafting[5-7]. These methods have different effects and only treat patients with early necrosis of femoral head. Therefore, a noninvasive alternative to surgery is important.

ESWT is a noninvasive treatment which has applied in varied diseases. And it also has implemented in orthopedics and traumatology for a period with a rapid development speed[8]. Nowadays, the application of ESWT in musculoskeletal disorders mainly included tendinopathies and bone defects[9]. In an animal 
experiment, ESWT may promote bone repair and increase bone mass in necrotic femoral head[10]. The first article about ESWT of ONFH published in 2001 and ESWT provided a potentially suitable alternative to invasive ways for treating ONFH in the author's opinion[8]. Many articles showed ESWT has an improvement in patients using the evaluation of HHS and VAS[12, 13]. And a case report even proved the efficiency of ESWT in patients with a stage of ARCO (Association Research Circulation Osseous) IV ,but more random control trail needs to be performed to prove it's efficacious in advanced disease [14].

To evaluate ESWT's effect on patients of ONFH and its long-term efficacy, we identified all suitable clinical trials from the electronic database and conducted this meta-analysis thoroughly to probe the influence of ESWT in HHS and VAS and the changes of lesion area of the femoral head on MRI.

\section{Methods}

\section{Study selection}

We selected Studies using PubMed, Embase, the Cochrane Library, CSPD, VIP, and CNKI databases from the inception dates to October 24, 2019 by two authors, without language restriction.खUsing

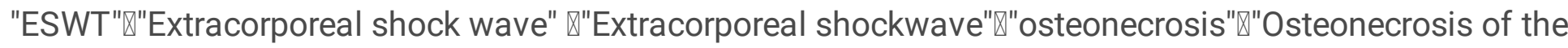

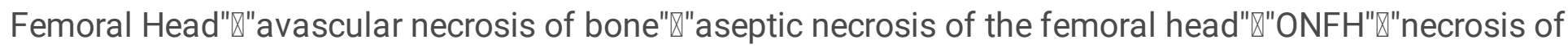
the femoral head" as the key words. According to different database retrieval formats, the keywords are combined with free words and Medical Subject Headings (MeSH) terms in the database for full-text searched, and the reference documents of the search literature are tracked.

\section{Inclusion criteria}

studies were included in this meta-analysis met following criteria $\mathbb{\nabla}(1)$ Studies using extracorporeal shock waves as an intervention with a control group; (2)studies object: a clear diagnosis of femoral head necrosis in radiology image or in MRI and there is no limited to gender, age, course of disease, etiology; (3)Outcome indicator $\ m$ main outcome indicator are HHS and VAS\secondary outcome indicator are progress of MRI.

\section{Exclusion criteria}

Exclusion criteria were as follows :(1) Review, case report, meetings abstracts, animal studies, editorial letters, guidance or comments; ( 2)No clear diagnostic criteria for disease;(3)Repeated studies; (4)incomplete data or data cannot be compared even after a calculation;(5)duration of follow-up less than six months;(6)sample size are less than 10 patients.

\section{Quality assessment}

We used the National Institute for Clinical Excellence (NICE) case series scoring standard to evaluate The case series study, and the case-control study was evaluated by the Newcastle-Ottawa Scale (NOS). We graded the random control trial (RCT) according to the modified jaded score. Four items were evaluated 
including the adequacy of the randomization process( 2 points), the adequacy of allocation concealment(2 points),double-blinding (2points) and withdrawals and dropouts( 2 points). Two researchers independently conducted a rigorous quality evaluation of the included literature by using the above scoring scale. When there were different views, the discussion was conducted with all researchers to solve the issue

\section{Data extraction}

Two researchers independently extracted the following information from each study: first author, year of publication, participant characteristics, study design, follow-up duration, total number of ONFH patients and hips, the average age of patients, the pulses and energy flux density of ESWT, and the risk factor. The clinical outcomes of our study included The HHS of patients before ESWT and after ESWT, The visual analogue scale pain score of patients before ESWT and after ESWT, rate of conversion to THA, The degree of radiographic progression,

\section{Statistical analysis}

We conducted this meta-analysis using Review Manager 5.3.Considering HHS, VAS and progress of imaging are all expressed by quantitative data, we used mean difference (MD) to analyze continuous outcomes with $95 \%$ confidence intervals $(\mathrm{Cl})$. When the parameter was measured with different ways or having a different unit of measurement, we used standard mean difference (SMD) to analyze continuous outcomes. Chi-squared tests and the $\mathrm{I}^{2}$ statistic were used to evaluate statistical heterogeneity. An $\mathrm{I}^{2}>$ $50 \%$ was considered that compared studies had significantly statistical heterogeneity and a subgroup analysis was carried out to find the cause of heterogeneity and then decided use random-effect or fixedeffect model. We performed egger test to evaluate publication bias. Values of $P<0.05$ were considered statistically significant. If the heterogeneity cannot be eliminated by subgroups analysis, a sensitive analysis will be preformed. We evaluated the publication bias with an egger test by using stata 15.

\section{Result}

\section{Literature search}

The process of the literature search is illustrated in Fig. 1, a total of 604 potentially relevant articles were identified from 6 databases. 311 duplicated articles were excluded by using Endnotes. After a title and abstract screened, 274 articles were excluded. 46 records considered potentially eligible and full text reviewed, but 37 were excluded for different reasons ( 27 articles lack of crucial data, 6 articles follow-up less than 6 months, and 4 articles sample sizes less than 10 cases). Finally, 9 articles were reflected in the present meta-analysis[15-23].

\section{Study characteristics}


The basic characteristics of included studies are shown in Table 1. The study included 409 patients with an average age of approximately43.24 (18-69)years, a pulse range from 800 to 6000, a female ratio of approximately $29.83 \%$, an energy flux density range from $0.12-0.51 \mathrm{~mJ} / \mathrm{mm}^{2}$ and an average follow-up of 54.3 (6-228) months. 9 studies included 1 RCT, 3 case control studies,and 5 case series studies. The RCT study was assessed according to modified jaded scale and got 4 points. 5 case series was applied in the NICE case series scoring criteria for quality evaluation and got an average point of 5.4. We used NOS scales to evaluate 3 case controls and got an average score of 8.2.

\begin{tabular}{|c|c|c|c|c|c|c|c|c|c|}
\hline included trai & year & study style & number/hips & wosen, no (\$) & mean age $(y)$ & treatment & pulses & energy flux density & follor up (months) \\
\hline Xing & 2003 & case seris & $69 / \mathrm{NA}$ & $17(254)$ & $41.9(19-56)$ & ESIT & 1000 & $0.18 \sim 0.25 \mathrm{aJ} / \mathrm{m} 2$ & 12 \\
\hline Vang et al & 2012 & case control & $\begin{array}{l}23 / 29 \\
25 / 28\end{array}$ & $5(10 \mathrm{~A})$ & $\begin{aligned} 39.8 & \pm 12.1(19-63) \\
39.9 & \pm 9.3(19-53)\end{aligned}$ & $\begin{array}{l}\text { ESNT } \\
C D+B G\end{array}$ & 6000 & $0.474 \mathrm{aJ} / \mathrm{m}$ & $\begin{array}{r}103.5 \pm 3.4(93-106) \\
104.5 \pm 4.3(95-108)\end{array}$ \\
\hline Cheng & 2015 & case series & $26 / 30$ & $10(356)$ & 43. 1 & ESNT & 1000 & $0.18 \sim 0.25 \mathrm{~mJ} / \mathrm{mm}$ & 12 \\
\hline Han et al & 2016 & case control & $\begin{array}{l}\mathrm{N} / 15 \\
\mathrm{X} / 15\end{array}$ & $6(324)$ & $\begin{array}{l}64.9 \pm 6.4 \\
63.8 \pm 6.2\end{array}$ & $\begin{array}{l}\text { ESIT } \\
\text { ESTI }\end{array}$ & $\begin{array}{l}1000 \\
1000\end{array}$ & $\begin{array}{l}0.12 \mathrm{~J} /=2 \\
0.32 \mathrm{~m} /=2\end{array}$ & $\begin{array}{l}6 \\
6\end{array}$ \\
\hline su & 2016 & case series & $30 / 38$ & $11(376)$ & $42.3(23-68)$ & ESVT & 1000 & $0.20 \sim 0.35 \mathrm{~mJ} / \mathrm{mm} 2$ & 16. $3(12-24)$ \\
\hline Fang et al & 2016 & BCT & $\begin{array}{l}10 / 16 \\
11 / 14 \\
12 / 12\end{array}$ & $10(304)$ & $\begin{array}{l}46.1 \pm 6.2(35-55) \\
40.5 \pm 9.3(29-60) \\
39.4 \pm 10.2(18-52)\end{array}$ & $\begin{array}{l}\text { ESVT } \\
\text { ESIT } \\
\text { ESIT }\end{array}$ & $\begin{array}{l}2000 \\
4000 \\
6000\end{array}$ & $\begin{array}{l}0.510 \mathrm{aJ} / \mathrm{m}^{2} \\
0.510 \mathrm{aJ} / \mathrm{m} 2 \\
0.510 \mathrm{aJ} / \mathrm{m} 2\end{array}$ & $\begin{array}{l}33.8 \pm 7.6(18-45) \\
30.0 \pm 7.1(18-44) \\
30.7 \pm 8.0(18-45)\end{array}$ \\
\hline $\mathrm{Tt}$ & 2017 & case control & $\begin{array}{l}69 / 79 \\
63 / 82\end{array}$ & $\begin{array}{l}22(326) \\
21(336)\end{array}$ & $\begin{array}{l}\text { 43. } 5 \pm 12.7(18-67) \\
\text { 44. } 8 \pm 14.4(18-69)\end{array}$ & $\begin{array}{l}\text { ESIT } \\
\text { CD }\end{array}$ & 800 & $\mathrm{NA}$ & $96(80-113)$ \\
\hline $\begin{array}{l}\text { Algarni et al } \\
\text { Xie }\end{array}$ & $\begin{array}{l}2018 \\
2018\end{array}$ & $\begin{array}{l}\text { case series } \\
\text { case series }\end{array}$ & $\begin{array}{l}21 / 33 \\
31 / 44\end{array}$ & $\begin{array}{l}12(574) \\
8(26 t)\end{array}$ & $\begin{array}{c}37.5 \pm 4.8 \quad(21-54) \\
41.2(22-60)\end{array}$ & $\begin{array}{l}\text { ESVT } \\
\text { ESIT }\end{array}$ & $\begin{array}{l}1500 \\
1000\end{array}$ & $\begin{array}{l}\mathrm{NH} \\
\mathrm{NA}\end{array}$ & $\begin{array}{c}60 \pm 42(24-108) \\
130.6(121-139)\end{array}$ \\
\hline
\end{tabular}

CD: core deconpression $\quad C D+B G$; core decompressiontbone grafting

\section{The HHS of patients before and after ESWT}

HHS scored patients according to four parts including pain, function, deformity and range of motion. More score patients got, the better function patients had. The pooled results of HHS with eight studies of 337 hips show that ESWT achieved higher Harris hip score after treatment (MD $=-20.03 ; 95 \% C l,-25.56$, -14.49;Fig.2). The differences were statistically significant( $(p<0.01)$. However, the heterogeneity analysis show an excessive heterogeneity $\left(l^{2}=91 \%\right)$.according to the language of articles, we divided articles into the Chinese group and the English group and performed a subgroups analysis(Fig 3). In subgroups analysis, the heterogeneity of the English group has been eliminated. ESWT also achieved higher scores compared to baseline scores.

\section{The VAS of patients before and after ESWT}

VAS is the level of pain expressed by eleven figures from 0 to 10.0 means no pain and 10 means excessive pain. Patients chose one figure to express their degree of pain. The pooled results of VAS with seven studies of 253 hips demonstrate that ESWT achieved lower VAS scores after treatment(MD= $2.77 ; 95 \% \mathrm{Cl}, 1.88,3.65 ; \mathrm{Fig} .4)$, the differences were statistically significant $(\mathrm{p}<0.01)$. However, the heterogeneity analysis shows an excessive heterogeneity $\left(\mathrm{I}^{2}=86 \%\right)$. We performed subgroups analysis(Fig 5). Studies were divided into the low score group and the high score group according to the degree of the 
pain score decreased. In subgroups analysis, the heterogeneity of low score group has been eliminated. All group of ESWT have achieved lower scores compared to baseline scores, the differences were statistically significant $(\mathrm{p}<0.01)$.

\section{Changes of lesion area on MRI before and after ESWT}

6 studies were include in this meta-analysis. We selected SMD as Consolidated statistics due to Different concepts of lesions in the 4 articles. The pooled results of lesion of MRI with 164 hips show that ESWT decreased the lesion area of MRI after treatment(SMD=1.03,Cl,0.75,1.30,Fig.6). the differences were statistically significant $(p<0.01)$. However, $\mathrm{Cl}$ of the four studies included 0 ,it can be referred that ESWT have no efficient effect on changing the lesion area of ONFH. the Heterogeneity analysis also show an Excessive heterogeneity $\left(l^{2}=97 \%\right)$.

\section{Effect of ESWT compare to surgical therapy in HHS and VAS}

Two studies compared ESWT to surgical method (core decompression or core decompression with bone grafting)in HHS and VAS[16,21]. We used degree of changed score as effect size to evaluate the effect of different intervention method. We conducted a calculation to get compared data by using raw data. The polled result of two studies of 180 patients(218hips)showed that ESWT have a better improvement in $\mathrm{HHS}(\mathrm{MD}=13.64 ; 95 \% \mathrm{Cl}, 10.60,16.65 ;$ Fig.7) compare to surgical therapy, the differences were statistically significant $(\mathrm{p}<0.01)$.And ESWT have a better effect on pain relief compare to surgical method(SMD $=1.25 ; 95 \% \mathrm{Cl}, 0.95,1.54 ;$ Fig.8).

\section{Etiologic factors}

A total of 5 studies (170 patients) recorded the etiologic factors and the number of patients. alcohol was the most common etiologic factor (66 patients, $39 \%$ ), followed by corticosteroid (43 patients, $25 \%$ )and idiopathic (42 patients, $25 \%$ ).

\section{Change of ARCO stage before and after ESWT}

ARCO staging is more systematic, more comprehensive and more practical compared to the previous staging of ONFH. Two articles recorded the ARCO stage of patients before and after ESWT. It is shown in Fig.9.Considering 3 patients were lost to follow-up, there is no significant change of ARCO stage before and after ESWT ( $p>0.05)$.

\section{Rate of total hip arthroplasty}

Three articles (104hips) recorded the rate of THA (total hip arthroplasty).About $19.2 \%$ hips developed a THA囚and $80.8 \%$ hips had survived approximately. The duration of follow-up reached 8-9years in one study with a rate of THA was $24 \%$.However,hip arthroplasty rate of surgical group (core decompression) reached to $64 \%[16]$. 


\section{Sensitive analysis}

In the comparison of VAS score before and after ESWT囚Heterogeneity $\left(I^{2}=54 \%\right)$ still existed in the high score group after a subgroups analysis (Fig 5). So a sensitive analysis was performed. When the study of Wu 2017 had been omitted, Heterogeneity $\left(I^{2}=24 \%\right)$ was significantly reduced. So we read this article again to find the reason. Relatively small number of pulses may be one reason for this heterogeneity. This article type is case-control and lack of blinding method may be another reason.

\section{publication bias}

We performed an egger test to evaluate the publication bias of the change of HHS sore (table 2). It can be referred that there is no publication bias by the $p$-value. The value of $p$ is 0.477 and more than 0.05 .

\begin{tabular}{r|rrrrrr}
\multicolumn{1}{l}{ Number of studies $=8$} & & & Root MSE & $=3.566$ \\
\hline Std_Eff & Coef. & Std. Err. & $t$ & P $>|t|$ & [958 Conf. Interval] \\
\hline slope & -.7269636 & 1.315246 & -0.55 & 0.600 & -3.945256 & 2.491329 \\
bias & -3.789236 & 4.998494 & -0.76 & 0.477 & -16.02011 & 8.441638 \\
\hline
\end{tabular}

Test of Ho: no small-study effects $\quad P=0.477$

table 2.publication bias

\section{Discussion}

In this meta-analysis, 9 studies with 409 patients were include. We evaluated the effect of ESWT on pain relieved and motion function by comparing the change of the HHS and the VAS. The lesion area on MRI before and after ESWT was compared and we also preformed a comparison on ESWT and surgical therapy (core decompression or core decompression with bone grafting).

Patient HHS had been considerably improved after ESWT. Following consideration of the heterogeneity, we performed a subgroup analysis to eliminate the heterogeneity. This subgroup analysis showed the difference between Chinese articles and English articles, it may cause by races. But no matter what the reason is, the HHS of each group of patients had been enhanced to different degrees after ESWT. ESWT also show powerful ability in decreasing score of VAS. But there are also exist the heterogeneity. It may be influenced by measurer's induction and personal subjective feelings. So we performed a subgroup analysis according to the degree of the score decreased. Heterogeneity $\left(I^{2}=54 \%\right)$ still existed in the high score group after a subgroups analysis. So a sensitive analysis was performed. When the study of Wu 2017 had been omitted, Heterogeneity $\left(I^{2}=24 \%\right)$ was significantly reduced. Relatively small number of pulses may be one of the reasons for this heterogeneity. This article type is case-control and lack of blinding method may be another factor. This outcome shows ESWT can reduce pain and improve motor function and range of motion. Compared to core decompression or core decompression with bone grafting, ESWT also had an advantage in pain relief and improvement of motor function. 
One article showed that ESWT may halt or delay the radiographic progression of the disease in the precollapse stage. However, there were no significant improvements in the lesion area of the femoral head on MRI in our meta-analysis, and there is no significant change in the ARCO stage before and after ESWT.

Compared to core decompression or core decompression with bone grafting, ESWT has an advantage on pain relieved and improvement of motor function in this meta-analysis. Italian experience on the use of ESWT for ONFH also showed it is an efficient method in initials stages of the disease and better than core decompression and bone grafting[24]. A combination treatment of alendronate, extracorporeal shock and hyperbaric oxygen may delay or discontinue the development of ONFH in patients[25].Another article showed a combination treatment of alendronate, extracorporeal shock and hyperbaric oxygen is effective for early hip necrosis comparable to ESWT in short-term follow-up. Further studies are necessary to prove the long-term effect of a combination treatment.

For the above etiologic factor of ONFH, complications of treating leukemia also included ONFH. ESWT also showed an efficient effect on ONFH causing by leukemia in the short term by resolving the suffering of bone marrow edema[26].

ESWT group had a lower rate to THA compared to the surgical group. Even in a long-term follow up,ESWT group also showed a low rate to THA. One of the articles showed low degree of ARCO had a lower rate of THA[19].And Vulpiani et al found $66 \%$ of ARCO III hips developed to THA at final follow-up[27].So ESWT may decrease the rate of THA in an early stage of ONFH.

ESWT has been reported to treat a variety of disorders effectively, such as diabetic feet, Erectile Dysfunction, Plantar Fasciitis, and ONFH, especially for ONFH[28-31]. The mechanism of ESWT in the treatment of femoral head necrosis is not completely clear[32],One hypothesis is that ESWT could induce microfracture to accelerate bone healing and increase pain threshold[13]. L, Z. et al. discovered moderate extracorporeal shock wave intensity can enhance MSCs ( mesenchymal stem cells) proliferation, inducing development of MSCs into osteoblasts, and prevented MSCs from converting adipocytes. This might be a mechanism of ESWT can treat ONFH[33]. ESWT obviously enhances angiogenic and osteogenic effects of the MSCs by means of the nitric oxide pathway[34]. Ma et al believed ESWT may increase blood supply and repair necrotic subchondral bone by reinforcing the expression of vascular endothelial growth factor[35]. More basic experiments on the mechanism of ESWT need to be carried out.

\section{Limitation}

Limitations exist in this meta-analysis. First, one study only had a 6 months follow up[17].Second囚only one RCT was included this meta-analysis, the quality of the included studies was poor accompanied a low level of evidence. In addition, the variance of populations, VAS, and the way of ESWT contributed to a high level of heterogeneity. So more rigorous RCT need to be done to identify the effect of ESWT compare to other treatment method. 


\section{Conclusion}

This meta-analysis suggests that ESWT improves motion function, range of motion and relieve pain in early stages of disease. And ESWT have a lower rate to THA compare to surgical group(core decompose or core decompression with bone grafting), but there is no significant change in the lesion area of the femoral head on MRI and stage change after ESWT.

\section{Abbreviations}

ESWT: extracorporeal shock wave therapy; ONFH: osteonecrosis of femoral head; AVNFH: avascular necrosis of the femoral head; ANFH aseptic necrosis of the femoral head; ARCO: Association Research Circulation Osseous; THA: Total hip arthroplasty; MD: mean difference; $\mathrm{Cl}$ :confidence intervals; SMD: standard mean difference; HHS: Harris hip score; VAS: visual analogue score; MeSH: Medical Subject Headings.

\section{Declarations}

\section{Competing interests}

The authors declare that they have no conflict of interests.

\section{Funding}

This research did not receive any funding.

\section{Availability of data and materials}

Data sharing is not applicable in the current study.

consent for publication

Written informed consent for publication was obtained from all participants.

\section{Authors' contributions}

The search strategy was developed by LLP and YG. LLP and YG completed the electronic search and selected appropriate literature. JM extracted the information of the selected studies. ZCJ gave advice for the data analysis and the result of analysis. JM completed the manuscript writing. All the authors carefully checked and approved the final manuscript.

\section{Ethics approval and consent to participate}

This article does not contain any studies with human participants or animals performed by any of the authors. 


\section{acknowledgements}

Not applicable

\section{References}

1. Microsurgery Department of the Orthopedics Branch of the Chinese Medical Doctor Association, Group from the Osteonecrosis and Bone Defect Branch of the Chinese Association of Reparative and Reconstructive Surgery, Microsurgery and Reconstructive Surgery Group of the Orthopedics Branch of the Chinese Medical Association, Chinese Guideline for the Diagnosis and Treatment of Osteonecrosis of the Femoral Head in Adults. J Orthopaedic surgery2017;9, 3-12.

2. Moya-Angeler $\mathrm{J}$,Gianakos AL, Villa JC. et al. Current concepts on osteonecrosis of the femoral head. J World journal of orthopedics 2015;6, 590-601.

3. Kubo T, Ueshima K, Saito M.et al. Clinical and basic research on steroid-induced osteonecrosis of the femoral head in Japan. J Journal of orthopaedic science 2016;21, 407-13.

4. Zalavras Charalampos G,Lieberman Jay R, Osteonecrosis of the femoral head: evaluation and treatment.[J] .J Am Acad Orthop Surg, 2014;22: 455-64.

5. Koo KH, Kim R, Ko GH, Song HR, Jeong ST, Cho SH. Preventing collapse in early osteonecrosis of the femoral head. A randomised clinical trial of core decompression. J Bone Joint Surg Br. 1995;77:8704.

6. Learmonth ID, Maloon S, Dall G. Core decompression for early atraumatic osteonecrosis of the femoral head. J Bone Joint Surg Br. 1990;72:387-90.

7. Saito S, Ohzono K, Ono K. Joint-preserving operations for idiopathic avascular necrosis of the femoral head. Results of core decompression, grafting and osteotomy. J Bone Joint Surg Br. 1988;70:78-84.

8. Thiel M,Nieswand M,Dörffel M, The use of shock waves in medicine-a tool of the modern OR: an overview of basic physical principles, history and research.[J] .Minim Invasive Ther Allied Technol, 2000;9: 247-53.

9. Saggini R,Di Stefano A,Saggini A et al. CLINICAL APPLICATION OF SHOCK WAVE THERAPY IN MUSCULOSKELETAL DISORDERS: PART I.[J] .J. Biol. Regul. Homeost. Agents, 2015;29: 533-45.

10. Ma Huan-Zhi,Zhou Dong-Sheng,Li Dong et al. A histomorphometric study of necrotic femoral head in rabbits treated with extracorporeal shock waves.[J] .J Phys Ther Sci,2017;29: 24-28.

11. Ludwig J,Lauber S,Lauber $\mathrm{H} \mathrm{J}$ et al. High-energy shock wave treatment of femoral head necrosis in adults.[J] .Clin. Orthop. Relat. Res.,2001;119-26.

12. Lee Jin-Young,Kwon Jae-Woo,Park Jung-Seob et al. Osteonecrosis of Femoral Head Treated with Extracorporeal Shock Wave Therapy: Analysis of Short-term Clinical Outcomes of Treatment with Radiologic Staging.[J] .Hip Pelvis,2015;27: 250-7. 
13. Gao Fuqiang,Sun Wei,Li Zirong et al. High-Energy Extracorporeal Shock Wave for Early Stage Osteonecrosis of the Femoral Head: A Single-Center Case Series.[J] .Evid Based Complement Alternat Med, 2015;468090.

14. Ma Yue Wen, Jiang Dong Lei, Zhang Dai et al. Radial Extracorporeal Shock Wave Therapy in a Person With Advanced Osteonecrosis of the Femoral Head.[J] .Am J Phys Med Rehabil, 2016;95: 133-9.

15. Xing Gengyan, Bai Xiaodong, Du Mingkui, et al. Efficacy of extracorporeal shock wave in the treatment of avascular necrosis of the femoral head in adults J. Chinese Journal of Physical Medicine and Rehabilitation.2003;28-30.

16. Wang CJ, Huang CC, Wang JW, et al. Long-term results of extracorporeal shockwave therapy and core decompression in osteonecrosis of the femoral head with eight- to nine-year follow-up. Biomed $J$ 2012;35, 481-5.

17. Cheng Yi, Li Ping, Efficacy analysis of ESWT in the treatment of avascular necrosis of the femoral head J. China Medical Science.2015;5, 20-26.

18. Wang CJ, Huang CC, Yip HK, et al. Dosage effects of extracorporeal shockwave therapy in early hip necrosis. Int J Surg 2016;35, 179-86.

19. Su Yi, Chen Yuyu, Yan Liang, et al.. Clinical observation of high-energy extracorporeal shock wave in the treatment of early femoral head necrosis J. Biotechnology World. 2016;87-8.

20. Han Y, Lee JK, Lee BY,et al. Correction: Effectiveness of Lower Energy Density Extracorporeal Shock Wave Therapy in the Early Stage of Avascular Necrosis of the Femoral Head. Ann Rehabil Med 2017;41, 337-8.

21. Wu Zheng, Feng Yangyang, Yin Yanbing. et al. Comparative study of long-term clinical efficacy of extracorporeal shock wave and core decompression for early femoral head necrosis J. Journal of Traumatic Surgery. 2017;19, 48-51.

22. Algarni AD, Al Moallem HM, Clinical and Radiological Outcomes of Extracorporeal Shock Wave Therapy in Early-Stage Femoral Head Osteonecrosis. Adv Orthop 2018;7410246.

23. Xie K, Mao Y, Qu X, et al.. High-energy extracorporeal shock wave therapy for nontraumatic osteonecrosis of the femoral head. Journal of Orthopaedic Surgery and Research2018,13.

24. Russo Sergio,Sadile Francesco,Esposito Roberto et al. Italian experience on use of E.S.W. therapy for avascular necrosis of femoral head.[J] .Int J Surg, 2015;24: 188-90.

25. Liu Tiansheng,Ma Jinchao,Su Bin et al. A 12-year follow-up study of combined treatment of postsevere acute respiratory syndrome patients with femoral head necrosis.[J] .Ther Clin Risk Manag, 2017;13: 1449-1454.

26. Te Winkel Mariël L, Pieters Rob, Wind Ernst-Jan D et al. Management and treatment of osteonecrosis in children and adolescents with acute lymphoblastic leukemia.[J] .Haematologica, 2014ه99: 430-6.

27. Vulpiani Maria Chiara, Vetrano Mario, Trischitta Donatella et al.Extracorporeal shock wave therapy in early osteonecrosis of the femoral head: prospective clinical study with long-term follow-up.[J] .Arch Orthop Trauma Surg, 2012;132: 499-508. 
28. Yang Jong-Phil, Lee Ye-Na, Son Ji Won et al. The Impact of Extracorporeal Shock Wave Therapy on Microcirculation in Diabetic Feet: A Pilot Study.[J] .Adv Skin Wound Care, 2019;32: 563-567.

29. Kim Kang Sup, Jeong Hyun Cheol, Choi Sae Woong et al. Electromagnetic Low-Intensity Extracorporeal Shock Wave Therapy in Patients with Erectile Dysfunction: A Sham-Controlled, Double-Blind, Randomized Prospective Study.[J] .World J Mens Health, 2019;14-356.

30. Xu Dingli, Jiang Weiyu, Huang Dichao et al. Comparison Between Extracorporeal Shock Wave Therapy and Local Corticosteroid Injection for Plantar Fasciitis.[J] .Foot Ankle Int, 2019; 12: 1071100719891111.

31. Tang Hua-Yu, Zhao Yu, Li Yu-Zhi et al. Effectiveness of extracorporeal shock wave monotherapy for avascular necrosis of femoral head: A systematic review protocol of randomized controlled trial.[J] .Medicine (Baltimore), 2019; 98: 15119.

32. Cheng Jai-Hong, Wang Ching-Jen, Biological mechanism of shockwave in bone.[J] .Int J Surg, 2015;24: 143-6.

33. Zhai Lei, Sun Nan, Zhang Bo et al. Effects of Focused Extracorporeal Shock Waves on Bone Marrow Mesenchymal Stem Cells in Patients with Avascular Necrosis of the Femoral Head.[J] .Ultrasound Med Biol,2016;42: 753-62.

34. Yin Tsung-Cheng, Wang Ching-Jen, Yang Kunder D et al. Shockwaves enhance the osteogenetic gene expression in marrow stromal cells from hips with osteonecrosis.[J] .Chang Gung Med J,2011; 34: 367-74.

35. Ma Huan-Zhi, Zeng Bing-Fang, Li Xiao-Lin, Upregulation of VEGF in subchondral bone of necrotic femoral heads in rabbits with use of extracorporeal shock waves.[J] .Calcif. Tissue Int., 2007;81: 12431.

\section{Figures}




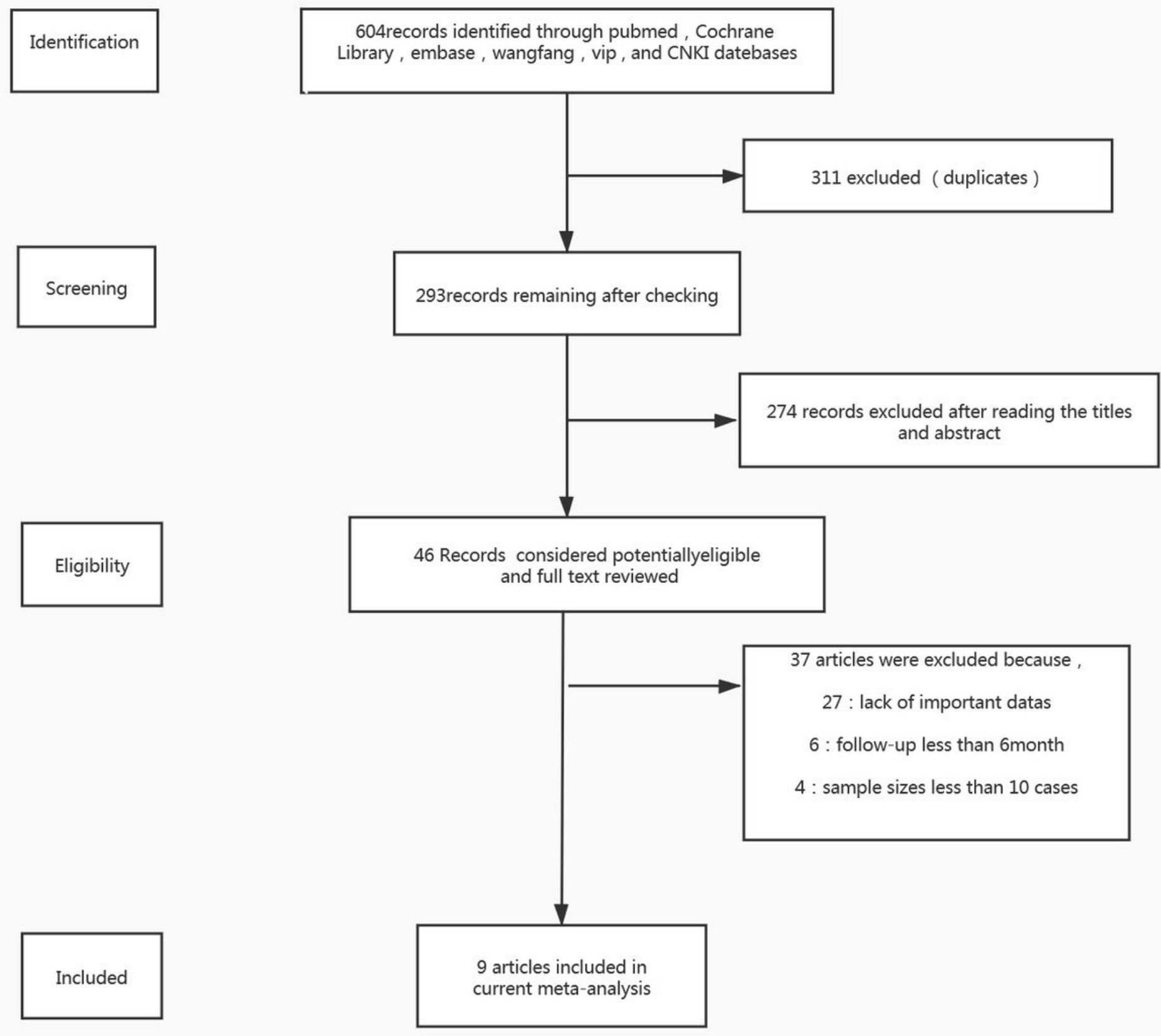

\section{Figure 1}

Flow chart of study selection, using PRISMA (Preferred Reporting Items for Systematic Meta-Analyses) guidelines. 


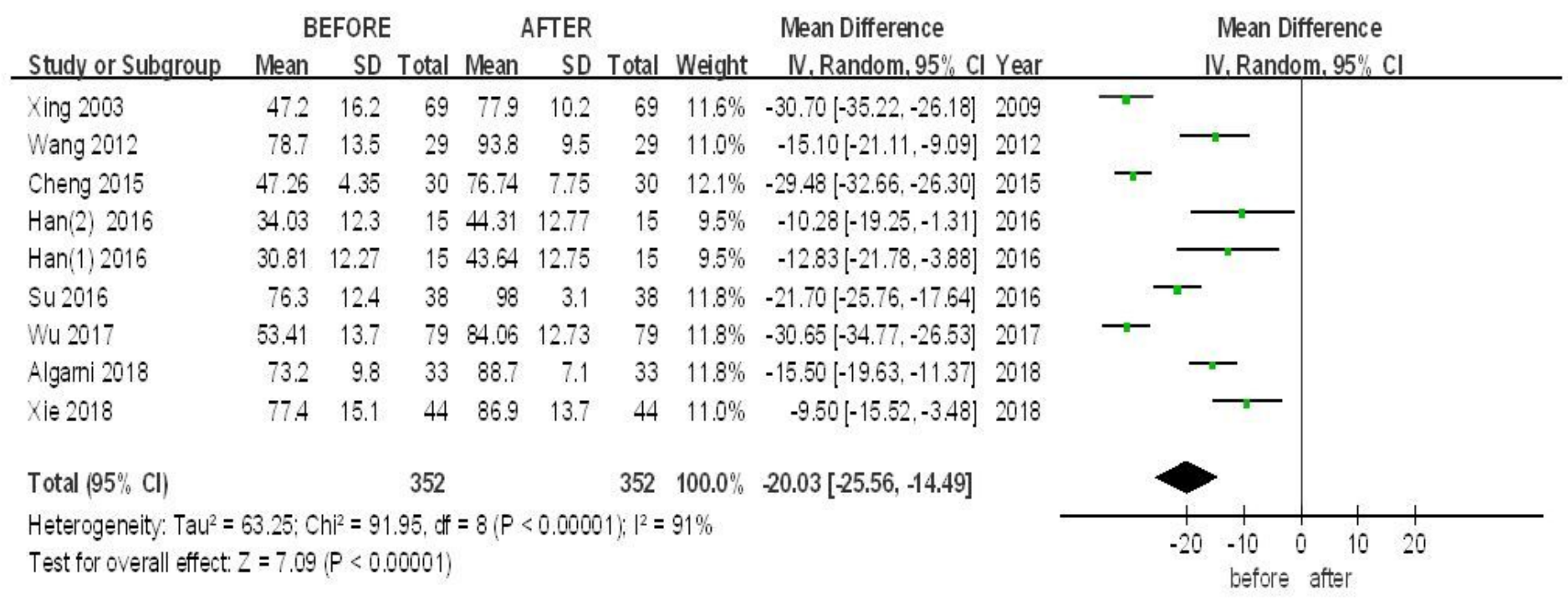

\section{Figure 2}

The change of HHS

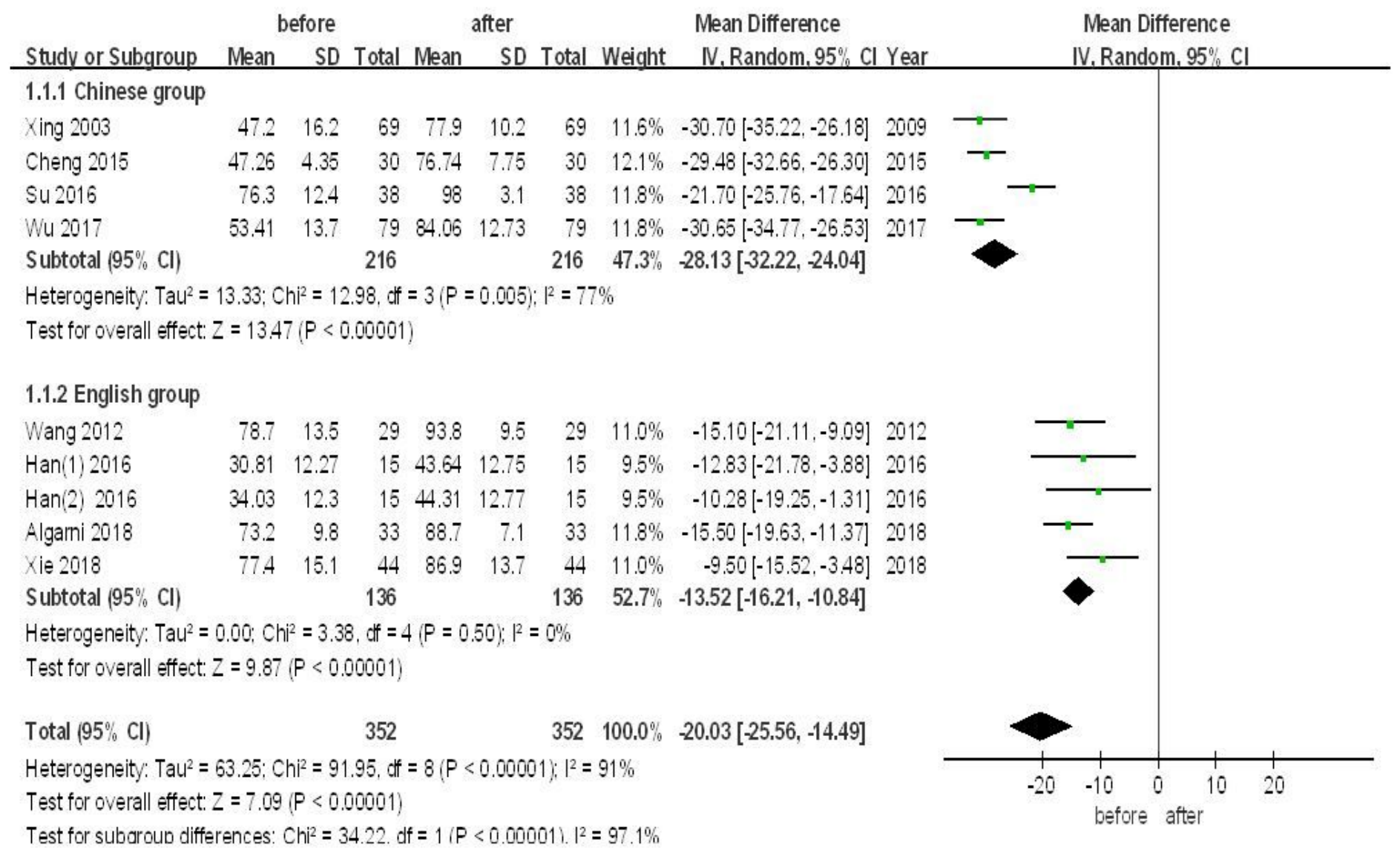

\section{Figure 3}

Subgroopanalysis of the change of HHS 
before after Mean Difference Mean Difference

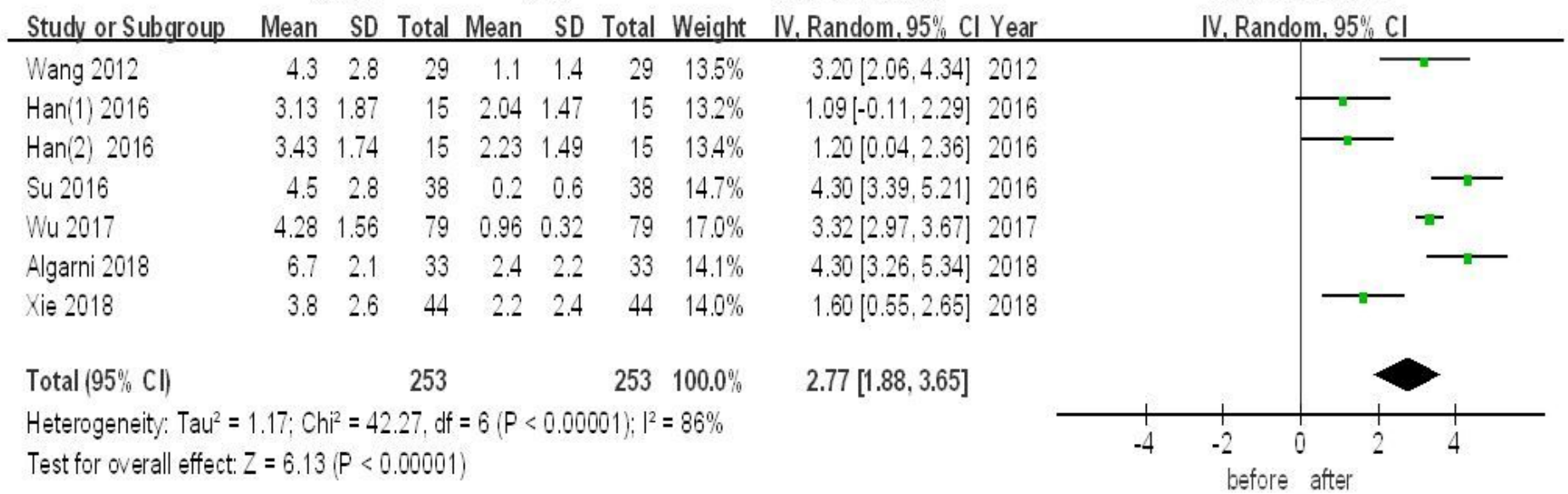

\section{Figure 4}

The change of VAS

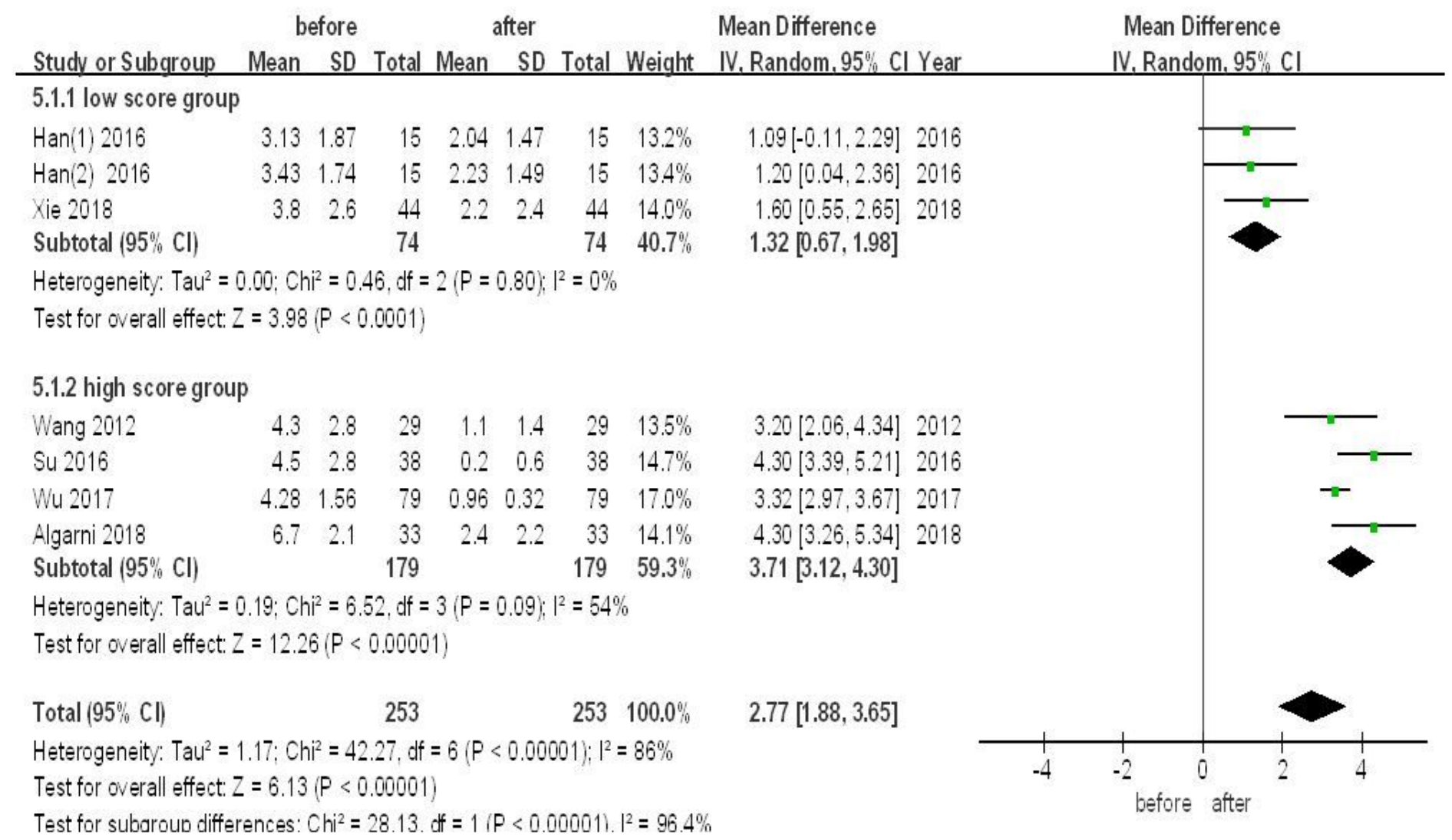

Figure 5

Subgroup analysis of the change of VAS 


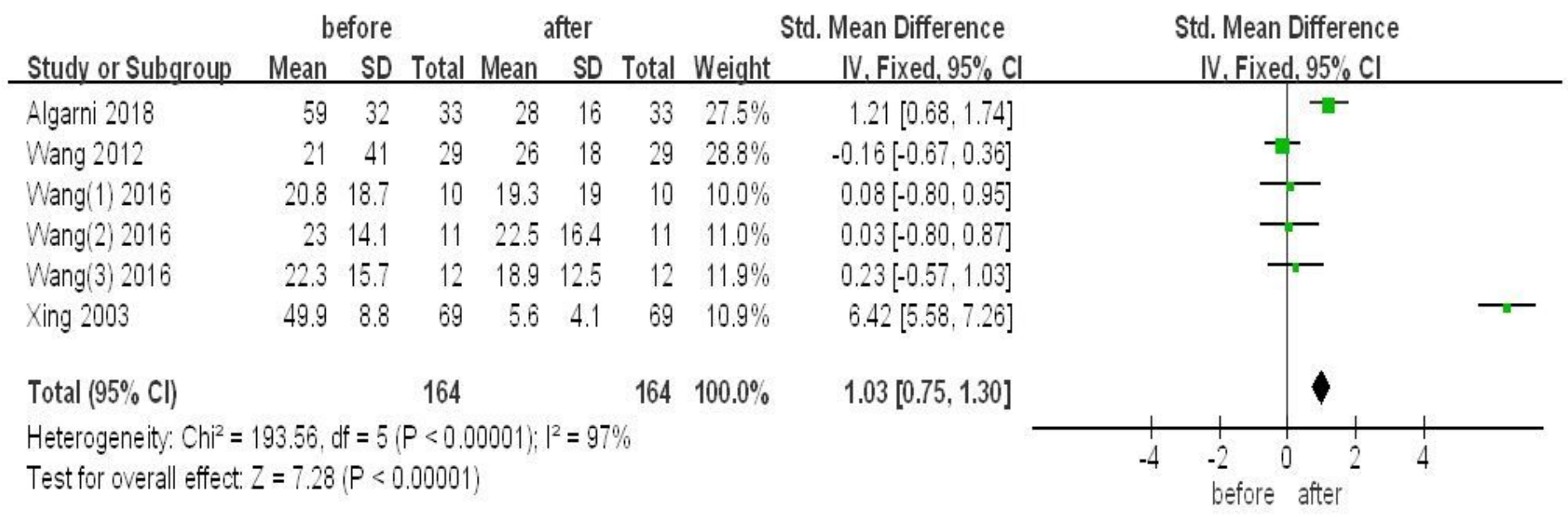

\section{Figure 6}

Changes of lesion area on MRI

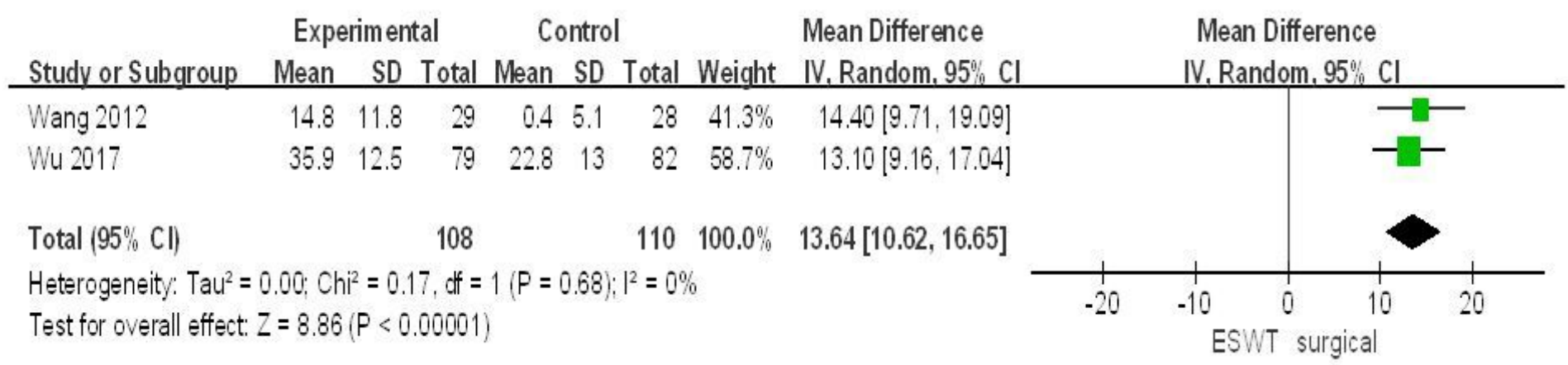

\section{Figure 7}

Effect of ESWT compare to surgical therapy in HHS

Experimental Control Std. Mean Difference Std. Mean Difference

Study or Subqroup Mean SD Total Mean SD Total Weight IV, Random, $95 \% \mathrm{Cl}$

IV. Random, $95 \% \mathrm{Cl}$

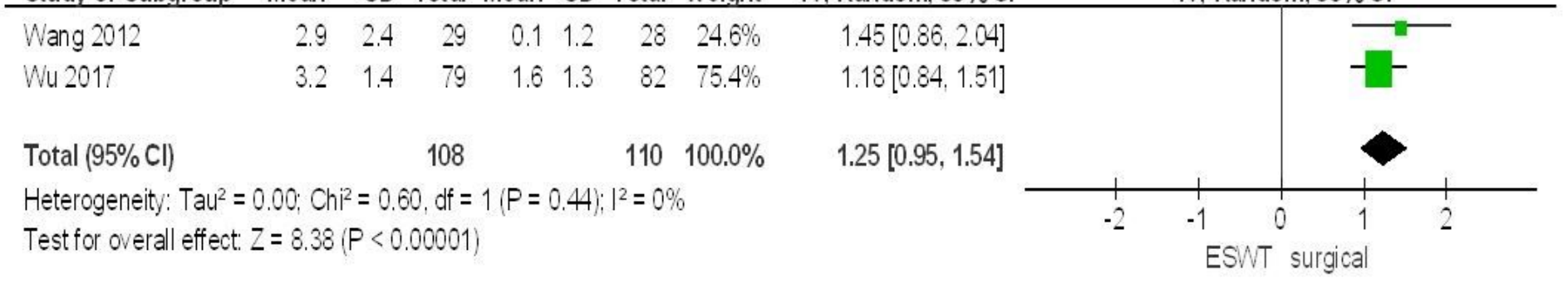

Figure 8

Effect of ESWT compare to surgical therapy in VAS 


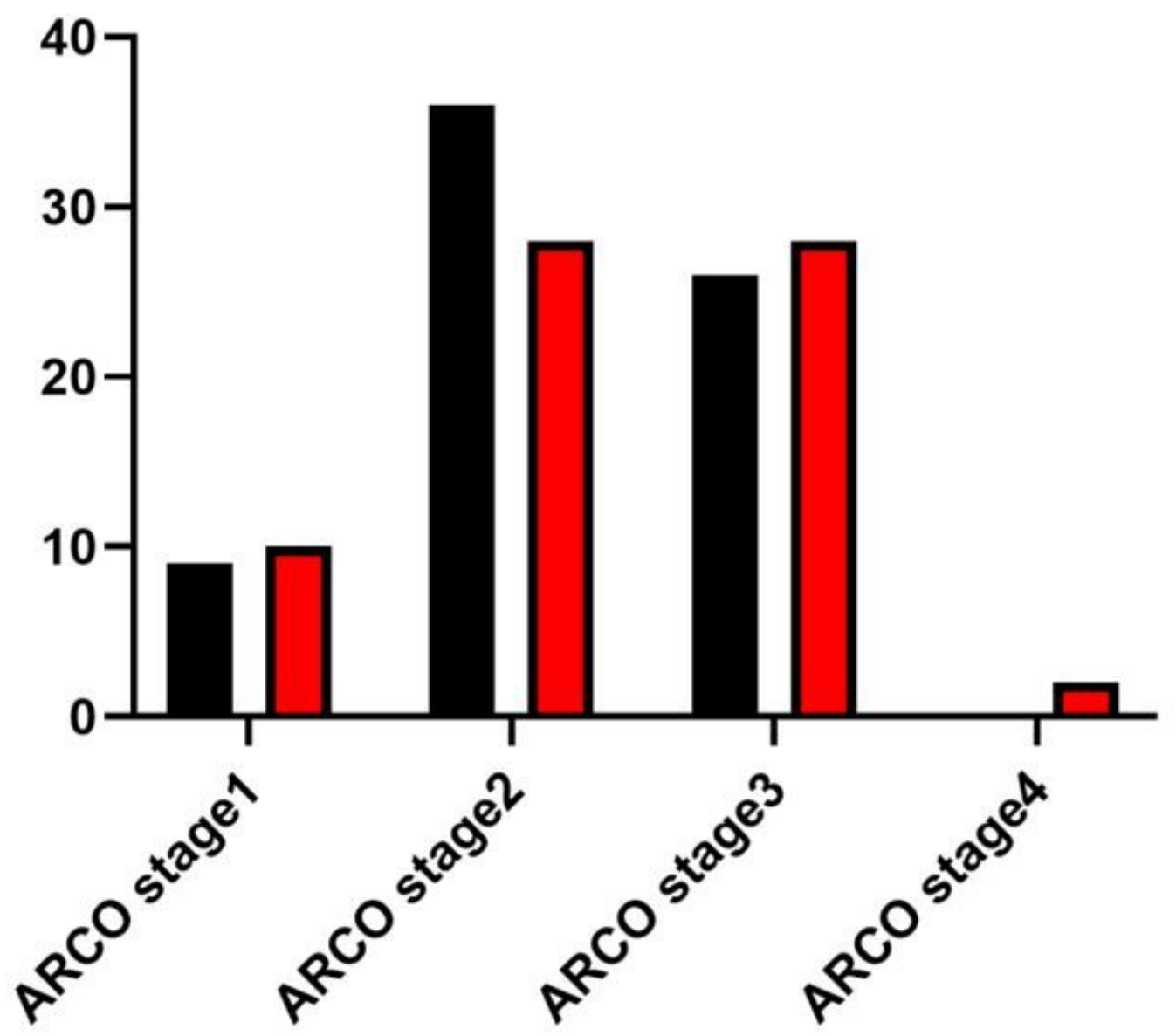

Figure 9

Change of ARCO stage 